Journal of Applied Analysis

Vol. 5, No. 1 (1999), pp. 119-123

\title{
NONLINEAR ALTERNATIVE: APPLICATION TO AN INTEGRAL EQUATION
}

\author{
A. CONSTANTIN \\ Received January 30, 1997 and, in revised form, July 21, 1998
}

\begin{abstract}
We prove the existence of solutions to an integral equation modeling the infiltration of a fluid in an isotropic homogeneous porous medium.
\end{abstract}

Let us consider the mathematical theory of the infiltration of a fluid from a cylindrical reservoir into an isotropic homogeneous porous medium. Describing this phenomena in the Boussinesq model (see [1]), we are led to the nonlinear integral equation (see also [5])

$$
u^{2}(t)=L(t)+\int_{0}^{t} K(t-s) u(s) d s, \quad t \in R_{+},
$$

where $K$ and $L$ are known smooth functions depending on physical parameters. The unknown function $u$ denotes the height of the percolating fluid above the horizontal base, multiplied by a positive factor. This is the reason why, from the physical point of view, nonnegative solutions of (1) are most interesting.

Our analysis of equation (1) is based on the following (see [3, 4]):

1991 Mathematics Subject Classification. 45 G.

Key words and phrases. Integral equation, nonlinear alternative.

ISSN 1425-6908 (c) Heldermann Verlag. 
Nonlinear alternative. Assume that $O$ is an open subset of a convex set $S$ in a Banach space. Let $A: \operatorname{cl}(O) \rightarrow S$ be a compact map and assume that $0 \in O$. Then either $A$ has a fixed point in $\operatorname{cl}(O)$ or there is a point $x \in \partial O$ and an $\lambda \in(0,1)$ such that $x=\lambda A x$.

We denoted by $\operatorname{cl}(O)$ and $\partial O$ the closure and respectively the boundary of the open set $O$.

Theorem. Assume that $K \in C^{1}\left(\mathbb{R}_{+}, \mathbb{R}_{+}\right)$is nondecreasing and that $L \in$ $C^{1}\left(\mathbb{R}_{+}, \mathbb{R}_{+}\right)$is nondecreasing with $L(0)=0$. Then (1) has a solution $u(t)$ on $\mathbb{R}_{+}$with $u(t) \geq 0$ for $t \in \mathbb{R}_{+}$.

Proof. Let us consider the integrodifferential equation

$$
x^{\prime}(t)=L^{\prime}(t)+K(0) w(x(t))+\int_{0}^{t} K^{\prime}(t-s) w(x(s)) d s, \quad t>0,
$$

with the initial condition

$$
x(0)=0,
$$

where $w \in C\left(\mathbb{R}, \mathbb{R}_{+}\right)$is the function

$$
w(x)= \begin{cases}\sqrt{x}, & \text { if } x \geq 0 \\ 0, & \text { if } x<0\end{cases}
$$

We shall establish a priori estimates, independent of $\lambda$, for the solutions of the family of problems $(0 \leq \lambda \leq 1)$

$$
\left\{\begin{array}{l}
x^{\prime}(t)=\lambda\left[L^{\prime}(t)+K(0) w(x(t))+\int_{0}^{t} K^{\prime}(t-s) w(x(s)) d s\right], \\
x(0)=0
\end{array}\right.
$$

considered on the interval $[0,1]$.

Let $D^{+} f$ denote the right upper Dini derivative of the function $f$.

If $x(t)$ is a solution to $(4 . \lambda)$ on $[0,1]$, let $y(t)=\sqrt{|x(t)|}, t \in[0,1]$. We have then that

$$
\left(D^{+} y^{2}\right)(t):=\limsup _{h \rightarrow 0^{+}} \frac{y^{2}(t+h)-y^{2}(t)}{h} \leq\left|x^{\prime}(t)\right|, \quad 0<t<1,
$$

as

$$
\frac{y^{2}(t+h)-y^{2}(t)}{h}=\frac{|x(t+h)|-|x(t)|}{h} \leq \frac{|x(t+h)-x(t)|}{h}, \quad 0<t<1 .
$$

Taking into account $(4 . \lambda)$, we obtain

$$
\left(D^{+} y^{2}\right)(t) \leq L^{\prime}(t)+K(0) y(t)+\int_{0}^{t} K^{\prime}(t-s) y(s) d s, \quad 0<t<1 .
$$


Let us now denote

$$
\theta(t):=L(t)+K(0) \int_{0}^{t} y(s) d s+\int_{0}^{t}\left\{\int_{0}^{s} K^{\prime}(s-\tau) y(\tau) d \tau\right\} d s, \quad 0 \leq t \leq 1 .
$$

By the above we have that

$$
\left(D^{+} y^{2}\right)(t) \leq \theta^{\prime}(t), \quad 0<t<1
$$

or, equivalently,

$$
\left(D^{+}\left(y^{2}-\theta\right)\right)(t) \leq 0, \quad 0<t<1 .
$$

We infer from this inequality by continuity that the function $\left(y^{2}-\theta\right)$ is nondecreasing (cf., e.g., [2]). Hence

$$
y^{2}(t)-\theta(t) \leq y^{2}(0)-\theta(0)=0, \quad 0 \leq t \leq 1,
$$

that is,

$$
y^{2}(t) \leq L(t)+K(0) \int_{0}^{t} y(s) d s+\int_{0}^{t}\left\{\int_{0}^{s} K^{\prime}(s-\tau) y(\tau) d \tau\right\} d s, \quad 0 \leq t \leq 1 .
$$

Let us define

$V(t)=1+L(1)+K(0) \int_{0}^{t} y(s) d s+\int_{0}^{t}\left\{\int_{0}^{s} K^{\prime}(s-\tau) y(\tau) d \tau\right\} d s, \quad 0 \leq t \leq 1$.

We have then that $1 \leq V(t)$ and $y^{2}(t) \leq V(t)$ on $[0,1]$.

Observe that

$$
V^{\prime}(t)=K(0) y(t)+\int_{0}^{t} K^{\prime}(t-s) y(s) d s, \quad 0 \leq t \leq 1,
$$

thus

$$
V^{\prime}(t) \leq K(0) \sqrt{V(t)}+\int_{0}^{t} K^{\prime}(t-s) \sqrt{V(s)} d s, \quad 0 \leq t \leq 1
$$

and since $V(t) \geq 1$ on $[0,1]$ is nondecreasing, we obtain that

$$
\frac{V^{\prime}(t)}{2 \sqrt{V(t)}} \leq \frac{1}{2} K(0)+\frac{1}{2} \int_{0}^{t} K^{\prime}(t-s) d s=\frac{1}{2} K(t), \quad 0 \leq t \leq 1 .
$$

Note that

$$
\sqrt{V(0)} \leq 1+L(1)
$$

and therefore an integration yields

$$
\sqrt{V(t)} \leq 1+L(1)+\frac{1}{2} \int_{0}^{t} K(s) d s, \quad 0 \leq t \leq 1 .
$$

Recall that $y^{2}(t) \leq V(t)$ on $[0,1]$ so that

$$
\sqrt{|x(t)|} \leq 1+L(1)+\frac{1}{2} \int_{0}^{t} K(s) d s .
$$

Put $P^{2}=1+L(1)+(1 / 2) \int_{0}^{1} K(s) d s, P>0$. 
Let us denote $\|v\|=\sup _{0 \leq t \leq 1}\{|v(t)|\}$ for $v \in C[0,1]$ and let $X$ be the Banach space $\left\{x \in C^{1}[0,1]: x(0)=0\right\}$ with the norm $\|x\|_{1}=\|x\|+\left\|x^{\prime}\right\|$ for $x \in X$.

Observe that the linear operator $\mathcal{D}: X \rightarrow C[0,1]$ defined by $\mathcal{D} x=x^{\prime}$ has a bounded inverse $\mathcal{D}^{-1}$.

Denote by $F: X \rightarrow C[0,1]$ the operator

$$
\left.F x(t)=L^{\prime}(t)+K(0) w(x(t))+\int_{0}^{t} K^{\prime}(t-s) w(x(s)) d s\right], \quad 0 \leq t \leq 1,
$$

and define $A: X \rightarrow X$ by $A x=\mathcal{D}^{-1} F x$ for $x \in X$. It is clear that $A$ is a compact operator.

Let us consider the open set

$$
O=\left\{x \in X:\|x\|_{1}<1+P+\sup _{0 \leq t \leq 1}\left\{L^{\prime}(t)\right\}+K(1) \sqrt{P}\right\} .
$$

By (5) we have that there is no point $x \in \partial O$ such that for some $\lambda \in(0,1)$ we have $x=\lambda A x$ (if $x \in X$ satisfies equation (4. $\lambda$ ) for some $\lambda \in(0,1)$, then $\|x\| \leq P$ and

$$
\begin{aligned}
\left\|x^{\prime}\right\| & \leq \sup _{0 \leq t \leq 1}\left\{L^{\prime}(t)\right\}+K(0) \sqrt{P}+\sqrt{P} \int_{0}^{1} K^{\prime}(1-s) d s \\
& \left.\leq \sup _{0 \leq t \leq 1}\left\{L^{\prime}(t)\right\}+K(1) \sqrt{P}\right) .
\end{aligned}
$$

We deduce by the Nonlinear alternative that $A$ has a fixed point in $\operatorname{cl}(O)$, that is, there is a solution $x(t)$ on $[0,1]$ to the problem $(2)-(3)$. Since $x(0)=0$ we obtain from the form of $(2)$ that $x(t) \geq 0$ on $[0,1]$ so that, if we let $u(t)=\sqrt{x(t)}, 0 \leq t \leq 1$, we have

$$
2 u(t) u^{\prime}(t)=L^{\prime}(t)+K(0) u(t)+\int_{0}^{t} K^{\prime}(t-s) u(s) d s, \quad 0 \leq t \leq 1,
$$

and an integration yields $(u(0)=0$ and $L(0)=0)$

$$
u^{2}(t)=L(t)+\int_{0}^{t} K(t-s) u(s) d s, \quad 0 \leq t \leq 1 .
$$

We proved that (1) has a solution $u(t)$ on $[0,1]$ with $u(t) \geq 0$ for all $t$ in the interval $[0,1]$.

Let

$$
x_{1}=\lim _{t \rightarrow 1} u^{2}(t)=u^{2}(1)
$$


As before, we show that the integrodifferential equation

$$
\begin{aligned}
z^{\prime}(t)= & L^{\prime}(t)+\int_{0}^{1} K^{\prime}(t-s) u(s) d s+K(0) w\left(z(t)+x_{1}\right) \\
& +\int_{1}^{t} K^{\prime}(t-s) w\left(z(s)+x_{1}\right) d s, \quad t \geq 1,
\end{aligned}
$$

with initial condition

$$
z(1)=0
$$

has a solution $z(t)$ on $[1,2]$ and that $z(t) \geq 0$ on $[1,2]$.

Let us extend $u(t)$ to $[0,2]$ by letting

$$
u(t)=\sqrt{z(t)+x_{1}}, \quad 0 \leq t \leq 2 .
$$

The so-defined $u$ is a solution of (1) on [0,2].

Continuing this way, we construct a solution of $(1)$ on $\mathbb{R}_{+}$which is nonnegative on $\mathbb{R}_{+}$.

The equation (1) was also considered in [5] under the assumption that the function $r \rightarrow L(r) / \sqrt{r}$ is nondecreasing and convex on $(0, \infty)$. We can easily find examples of functions $L$ such that to the corresponding equation (1) our theorem is applicable and the results of [5] are not.

Acknowledgements. The author is grateful to the referees for the constructive recommandations that improved the initial version of this paper.

\section{References}

[1] Bear, J., Dynamics of Fluids in Porous Media, American Elsevier Pub. Co., New York, 1972.

[2] Bruckner, A., Differentiation of Real Functions, Lecture Notes in Math. 659, Springer-Verlag, Berlin-Heidelberg-New York, 1978.

[3] Dugundji, J. and Granas, A., Fixed Point Theory, PWN, Warszawa, 1982.

[4] Granas, A., Sur la méthode de continuité de Poincaré, C. R. Acad. Sci. Paris Ser. I Math. 282 (1976), 983-985.

[5] Okrasinśki, W., On a non-linear convolution equation occuring in the theory of water percolation, Ann. Polon. Math. 37 (1980), 223-229.

AdRian Constantin

Mathematisches Institut

UNIVERSITÄT ZÜRICH

WINTERTHURERSTRASSE 190, ZÜRICH

CH-8057, Switzerland 